\title{
Adverse Events in Primary Percutaneous Coronary Angioplasty With Drug-Eluting Stents Compared With Drug-Coated Balloons: A Retrospective Outlook
}

Hamid Sharif Khan ${ }^{1}$, Jahanzeb Malik ${ }^{1}$, Muhammad Mohsin ${ }^{1}$, Asim Javed ${ }^{1}$, Muhammad Umar Faroog

1. Cardiology, Rawalpindi Institute of Cardiology, Rawalpindi, PAK

Corresponding author: Jahanzeb Malik, heartdoc86@gmail.com

\section{Abstract \\ Objective}

To compare drug-eluting stents (DES) with drug-coated balloons (DCBs) in terms of major adverse cardiovascular and cerebrovascular events (MACCE) in patients who have undergone primary percutaneous coronary intervention (PPCI).

\section{Methodology}

Out of 210 angioplasties in six months, 80 patients were included; 40 in DES and 40 in DCB, respectively. All had a successful PPCI. It was defined as the achievement of thrombolysis in myocardial infarction (TIMI) grade II/III with $<20 \%$ residual stenosis for the DES and TIMI grade II/III with $<30 \%$ residual stenosis for the DCB. Any subsequent MACCE during the ensuing six months were assessed from emergency/outpatient records during their subsequent hospital visits, hospital registry, and telephonic interviews.

\section{Results}

The mean age in the DES group was $54.83+8.72$ years while it was $56.8+8.9$ years in the DCB group. The left anterior descending artery (LAD) was the culprit artery in the majority of the cases in both groups. The mean diameter of DES and DCB was $3.17+0.38 \mathrm{~mm}$ and $2.75+0.53 \mathrm{~mm}$, respectively. Overall, 15 adverse events were seen in the DES group and 16 in the DCB group. The difference was insignificant $(\mathrm{P}$-value $=\geqslant 0.999)$. There were nine hospitalizations due to chest pain in the DES group and eight in the DCB group. Total vessel revascularization (TVR) was seen in two patients in the DES group and three patients in the DCB group. None of the patients suffered a stroke. All variables of MACCE were non-significant (P-value $=\geqslant 0.999$ ).

\section{Conclusion}

Received 05/21/2020

DCB appears to be non-inferior to DES in PPCI at a median follow-up of six months.

Categories: Cardiology

Keywords: stemi, macce, des, dcb

\section{Introduction}

Acute myocardial infarction (AMI) is caused by the occlusion of a coronary artery due to the rupture or erosion of a plaque with thrombus formation [1]. Primary percutaneous coronary intervention (PPCI) is the standard treatment of choice in AMI patients presenting within 12 hours of the onset of chest pain [2]. Drugeluting stents (DES) are implanted in the blocked arteries after the restoration of thrombolysis in myocardial infarction (TIMI) flow during PPCI [3]. Various large trials have proven DES to reduce repeat revascularization, however, they do not reduce mortality as compared to balloon angioplasty alone and it increases the long-term risk of stent thrombosis and stent restenosis [4-5].

The drug-coated balloon (DCB) has emerged as an alternative treatment option to treat coronary artery stenosis [6]. DCBs are semi-compliant balloons with a chemotherapeutic, anti-proliferative drug (commonly paclitaxel) incorporated with an excipient to facilitate the drug transfer upon inflation of the balloon [7]. The presence of drugs with antiproliferative properties helps reduce the potential risk of an inflammatory response to a metal platform seen in cases of stents, thus reducing the risk of stent restenosis and stent thrombosis [8].

Compared to DES, coronary artery intervention with a DCB is a cumbersome procedure involving proper lesion preparation with pre-dilatation of the vessel with at least equal size as the semi-compliant balloon [9]. Special care is needed in the handling of DCB to avoid the dissipation of the antiproliferative drug before 
positioning it at the lesion. Prolonged inflation (usually 60 seconds or more) at a nominal pressure is advised to ensure adequate drug delivery at the lesion and ensuring there is no edge dissection, which may require stenting as a bailout if it limits blood flow in the coronary artery [10].

Several studies have demonstrated DCB to infer benefits during elective angioplasties of side branch occlusions [10-11]. Data are limited in patients with AMI. In this study, we sought to demonstrate the major adverse cardiovascular and cerebrovascular events (MACCE) among AMI patients undergoing PPCI with DES and DCB as a comparison in a tertiary cardiac center of Pakistan with a median follow-up of six months.

\section{Materials And Methods}

In this retrospective observational study, data collection was started after approval from the research and ethics review committee, Rawalpindi Institute of Cardiology. Records of patients were extracted from the inhouse cath-recording system from July 2019 to December 2019, a total period of six months.

All patients were preloaded with aspirin $300 \mathrm{mg}$ and clopidogrel $600 \mathrm{mg}$. Inclusion criteria were a successful PPCI procedure either with a DES (Xience Expedition, Abbott Laboratories, Abbott Park, Illinois) or a DCB (SeQuent Neo, B. Braun, Melsungen, Germany) at the discretion of the primary operator. Successful PPCI was defined as an achievement of TIMI grade II/III flow with $<20 \%$ residual stenosis for the DES group and TIMI grade II/III flow with $<30 \%$ residual stenosis for the DCB group. Both groups were started on aspirin 75 $\mathrm{mg}$ once daily and clopidogrel $75 \mathrm{mg}$ once daily. Their risk factors and baseline parameters were noted from their hospital records. Patients who were lost to follow-up or non-compliant to treatment and patients who were deferred stenting were excluded. Patients with comorbidities like diabetes, hypertension, and chronic kidney disease were also excluded.

Any subsequent MACCE including a history of stroke or transient ischemic attack (TIA), death due to AMI, re-infarction, target vessel revascularization (TVR), or hospital admission due to acute coronary syndrome (ACS) during the ensuing six months were assessed from the emergency/outpatient records during their subsequent hospital visits, hospital registry, and telephonic interviews.

Statistical Package for Social Sciences (SPSS) version 26 (IBM Corp., Armonk, NY) was used for data analysis. Continuous variables were expressed as mean \pm standard deviation (SD) and categorical variables as a percentage. The chi-square with the Fisher exact test was carried out for a comparison of categorical variables, respectively. P-value of less than 0.05 was considered significant.

\section{Results}

There were a total of 210 angioplasties done in six months. Out of these, 130 patients were excluded based on comorbid conditions, lost to follow-up, or non-compliance to dual antiplatelet therapy (DAPT). Eighty patients were included with 40 who had a DES in place and 40 had DCB angioplasty done. The baseline characteristics and patient demographics are shown in Table 1.

\begin{tabular}{|c|c|c|}
\hline Baseline characteristics & DES group & DCB group \\
\hline Age & 54.83 yrs +8.72 & 56.8 yrs +8.9 \\
\hline Gender & Male: $77.5 \%(n=31)$ Female: $22.5 \%(n=09)$ & Male: $72.5 \%(n=29)$ Female: $27.5 \%(n=11)$ \\
\hline Culprit artery & $\begin{array}{l}\text { LAD: } 52.5 \%(n=21) \text { RCA: } 22.5 \%(n=09) \text { LCX: } \\
25.0 \%(n=10)\end{array}$ & $\begin{array}{l}\text { LAD: } 52.5 \%(n=21) \text { RCA: } 25.0 \%(n=10) \text { LCX: } \\
22.5 \%(n=09)\end{array}$ \\
\hline Overall CAD & $\begin{array}{l}\text { SVCAD: } 35.0 \%(n=14) \text { DVCAD: } 52.5 \%(n=21) \\
\text { TVCAD: } 12.5 \%(n=05)\end{array}$ & $\begin{array}{l}\text { SVCAD: } 27.5 \%(n=11) \text { DVCAD: } 55.0 \%(n=22) \\
\text { TVCAD: } 17.5 \%(n=07)\end{array}$ \\
\hline $\begin{array}{l}\text { Multi-vessel disease: Involvement of more than } \\
\text { two coronary arteries }\end{array}$ & $65.0 \%(n=26)$ & $2.5 \%(n=29)$ \\
\hline Mean stent/balloon diameter & $3.17+0.38 \mathrm{~mm}$ & $2.75+0.53 \mathrm{~mm}$ \\
\hline
\end{tabular}

\section{TABLE 1: Demographics and baseline characteristics}

Drug-eluting stent (DES), drug-coated balloon (DCB), left anterior descending artery (LAD), left circumflex artery (LCX), right coronary artery (RCA), single-vessel coronary artery disease (SVCAD), double-vessel coronary artery disease (DVCAD), triple-vessel coronary artery disease (TVCAD), coronary artery disease (CAD) 


\section{Cureus}

\begin{tabular}{|c|c|c|}
\hline MACCE Component & DES Group & DCB Group \\
\hline Death & $2.5 \%(n=01)$ & $5.0 \%(n=02)$ \\
\hline Hospitalization due to ACS & $22.5 \%(n=09)$ & $20.0 \%(n=08)$ \\
\hline Target Vessel Revascularization (TVR) & $5.0 \%(n=02)$ & $7.5 \%(n=03)$ \\
\hline AMI (including stent thrombosis) & $7.5 \%(n=03)$ & $7.5 \%(n=03)$ \\
\hline Overall MACCE & $37.5 \%(n=15)$ & $40.0 \%(n=16)$ \\
\hline
\end{tabular}

\section{TABLE 2: Comparison of MACCE in the two subgroups at six months}

Acute coronary syndrome (ACS), acute myocardial infarction (AMI), target vessel revascularization (TVR), major adverse cardiovascular and cerebrovascular events (MACCE), drug-eluting stent (DES), drug-coated balloon (DCB)

Overall, 15 adverse events were recorded in the DES group and 16 in the DCB group. When the individual MACCE components were compared in the two groups, the difference was found to be clinically insignificant. None of the patients suffered a stroke. Overall, MACCE was insignificant between the two groups.

\section{Discussion}

PPCI with a DES is considered the treatment of choice in patients with AMI [2]. Stenting with a DES reduces target vessel revascularization (TVR) but there is no benefit in aborting recurrent AMI [4]. This was shown in a study that demonstrated an increased incidence of stent thrombosis among AMI patients treated with DES as compared with balloon angioplasty alone, thus paving a potential role of a DCB in PPCI [5]. The presence of a drug on the semi-compliant DCB provides a potential advantage in reducing the potential risk of in-stent restenosis or stent thrombosis [12].

Patients presenting with AMI usually have disease involving more than one coronary artery as demonstrated by a study in which $60 \%$ of patients had multivessel disease [13]. Similarly, in our study, about two-thirds of the patients had the involvement of more than one coronary artery, with a prevalence of $65.0 \%$ and $72.5 \%$ in the DES and DCB groups, respectively.

Several local studies have been conducted over the last few years on DCB but mostly they have been in the form of either a registry or a cohort with a small sample size [14]. Several studies showed the efficacy of DCB in various subsets of coronary artery lesions [15]. But the literature on AMI is limited as far as DCB is concerned.

This was a pilot study done in Pakistan, comparing the short-term outcomes in the form of MACCE among AMI patients treated with DES and DCB. As seen in our study, the overall MACCE at six months was similar in the two groups, with a P-value of $>0.818$. Similar results were seen in a study that found PPCI with DCB to be a safe and feasible option and considered non-inferior to DES in the AMI subset of patients [16]. The Drug-Eluting Baloon In Acute Myocardial Infarction (DEB-AMI) trial found no difference in MACCE at six months among patients treated with DCB only PPCI when compared with bare-metal stent (BMS) only PPCI [17]. The main reason for the similar MACCE was the timely management of the patients and the ability to achieve TIMI III blood flow in both the subgroups.

In our study, individual components of MACCE were not significantly different in the two groups. Death was seen in $5.0 \%$ and $2.5 \%$ of the DCB and DES groups, respectively. Similarly, the incidence of re-infarct at six months was homogeneous in the two groups, at $7.5 \%$ each. Related results were seen in a trial that showed a 30-day mortality of $2.4 \%$ in the DCB subgroup [18].

The incidence of TVR was seen as 7.5\% in the DCB group and 5.0\% in the DES group. The difference was, however, not significant. In one study, TVR at 90 days was found to be $3.3 \%$ with a DCB [19]. It is lower as compared to our study because we calculated the risk at six months. Being a non-randomized, single-center study, it has the limitations of small sample size and median time frame to follow-up. Further multicentered randomized control trials are needed to assess the efficacy of DCB in patients of AMI.

\section{Conclusions}

DCB appears to be non-inferior to DES in AMI patients undergoing PPCI at six months. The difference in 
MACCE between the two groups is insignificant in terms of death, target vessel revascularization, stroke, reinfarction, and hospitalization. DCB appears to be safe at a median follow-up of six months.

\section{Additional Information \\ Disclosures}

Human subjects: Consent was obtained by all participants in this study. Research and ethics review committee, Rawalpindi Institute of Cardiology issued approval RIC/DCA/19/20. It is certified that Dr Hamid Sharif, Consultant Cardiologist, had submitted a research proposal titled "A Retrospective Outlook at the Adverse Events In Primary Percutaneous Coronary Angioplasty with Drug-eluting Stents Compared With Drug-Coated Balloons" for approval and acceptance. The research proposal fulfilled the National/International standards/requirements. The research and ethics review committee has no objection and therefore approved and accepted the research proposal. Animal subjects: All authors have confirmed that this study did not involve animal subjects or tissue. Conflicts of interest: In compliance with the ICMJE uniform disclosure form, all authors declare the following: Payment/services info: All authors have declared that no financial support was received from any organization for the submitted work. Financial relationships: All authors have declared that they have no financial relationships at present or within the previous three years with any organizations that might have an interest in the submitted work. Other relationships: All authors have declared that there are no other relationships or activities that could appear to have influenced the submitted work.

\section{References}

1. Sakaguchi M, Ehara S, Hasegawa T, Matsumoto K, Nishimura S, Yoshikawa J, Shimada K: Coronary plaque rupture with subsequent thrombosis typifies the culprit lesion of non-ST-segment-elevation myocardial infarction, not unstable angina: non-ST-segment-elevation acute coronary syndrome study. Heart Vessels. 2017, 32:241-251. 10.1007/s00380-016-0862-6

2. Baber U, Mehran R, Giustino G, et al.: Coronary thrombosis and major Bleeding after PCI with drug-eluting stents: risk scores from Paris. J Am Coll Cardiol. 2016, 67:2224-2234. 10.1016/j.jacc.2016.02.064

3. Sarno G, Lagerqvist B, Nilsson J, et al.: Stent thrombosis in new-generation drug-eluting stents in patients with STEMI undergoing primary PCI: a report from SCAAR. J Am Coll Cardiol. 2014, 64:16-24. 10.1016/j.jacc.2014.04.022

4. Kedhi E, Joesoef KS, McFadden E, et al.: Second-generation everolimus-eluting and paclitaxel-eluting stents in real-life practice (COMPARE): a randomised trial. Lancet. 2010, 375:201-209. 10.1016/S01406736(09)62127-9

5. Wang X, Chen X, Sun W, et al.: Very late stent thrombosis in drug-eluting stents new observations and clinical implications. Cardiol Rev. 2019, 27:279-285. 10.1097/CRD.0000000000000283

6. Weissler EH, Zepel L, Greiner M, et al.: No increase in all-cause mortality at 2 years among patients undergoing drug-coated balloon angioplasty. JACC Cardiovasc Interv. 2020, 13:902-904. 10.1016/j.jcin.2020.01.197

7. Long CA, Zepel L, Greiner MA, Hammill BG, Patel MR, Jones WS: Use and 1-year outcomes with conventional and drug-coated balloon angioplasty in patients with lower extremity peripheral artery disease. Am Heart J. 2019, 217:42-51. 10.1016/j.ahj.2019.07.014

8. Nestelberger T, Kaiser C, Jeger R: Drug-coated balloons in cardiovascular disease: benefits, challenges, and clinical applications. Expert Opin Drug Deliv. 2020, 17:201-211. 10.1080/17425247.2020.1714590

9. Bonaventura K, Schwefer M, Yusof AKM, et al.: Systematic scoring balloon lesion preparation for drugcoated balloon angioplasty in clinical routine: results of the PASSWORD observational study. Adv Ther. 2020, 37:2210-2223. 10.1007/s12325-020-01320-2

10. Nugteren M, Serné A, Verhoeven B, et al.: Midterm outcomes of drug-coated balloon angioplasty in femoropopliteal lesions in a daily practice cohort. Ann Vasc Surg. 2020, 64:188-197. 10.1016/j.avsg.2019.09.032

11. Wang X, Lu W, Wang X, et al.: Drug-coated balloon angioplasty: predicting outcomes based on different patterns of drug-eluting stent restenosis. Int J Cardiovasc Imaging. 2020, 36:171-178. 10.1007/s10554-01901681-y

12. Tian J, Tang YD, Qiao S, et al.: Two-year follow-up of a randomized multicenter study comparing a drugcoated balloon with a drug-eluting stent in native small coronary vessels: the RESTORE Small Vessel Disease China trial. Catheter Cardiovasc Interv. 2020, 95:587-597. 10.1002/ccd.28705

13. Jahic E: Experience and outcomes of primary percutaneous coronary intervention for patients with STsegment elevation myocardial infarction of tertiary care center in Bosnia and Herzegovina. Med Arch. 2017, 71:183-187. 10.5455/medarh.2017.71.183-187

14. Caradu C, Lakhlifi E, Colacchio EC, Midy D, Bérard X, Poirier M, Ducasse E: Systematic review and updated meta-analysis of the use of drug-coated balloon angioplasty versus plain old balloon angioplasty for femoropopliteal arterial disease. J Vasc Surg. 2019, 70:981-995.e10. 10.1016/j.jvs.2019.01.080

15. Swamy AJ, Kumar A, Keshavamurthy G, Chadha DS: Drug-eluting balloon: Initial experience in patients with in-stent restenosis (ISR). Med J Armed Forces India. 2014, 70:243-246. 10.1016/j.mjafi.2014.01.001

16. Harada Y, Colleran R, Pinieck S, et al.: Angiographic and clinical outcomes of patients treated with drugcoated balloon angioplasty for in-stent restenosis after coronary bifurcation stenting with a two-stent technique. EuroIntervention. 2017, 12:2132-2139. 10.4244/EIJ-D-16-00226

17. Sato A, Kijima M, Ichimura S, et al.: Short-term outcome of percutaneous coronary intervention with directional coronary atherectomy followed by drug-coated balloon: a preliminary report. Cardiovasc Interv Ther. 2019, 34:149-154. 10.1007/s12928-018-0537-6

18. Cortese B, Buccheri D, Piraino D, Silva-Orrego P, Seregni R: Drug-coated balloon angioplasty: an intriguing 


\section{Cureus}

alternative for the treatment of coronary chronic total occlusions. Int J Cardiol. 2015, 187:238-239.

10.1016/j.ijcard.2015.03.223

19. Akutsu N, Ogaku A, Koyama Y, et al.: Effect of drug-coated balloon angioplasty on in-stent restenotic coronary lesions analyzed with optical coherence tomography and serial coronary artery angioscopy. Heart Vessels. 2019, 34:1925-1935. 10.1007/s00380-019-01447-5 